\title{
Essais
}

Revue interdisciplinaire d'Humanités

\section{Pluralité linguistique en classe de français langue seconde : quid de la variation?}

Des représentations aux attitudes des enseignant.e.s

\section{Marine Totozani et Sandra Tomc}

\section{(2) OpenEdition}

\section{Journals}

Édition électronique

URL : http://journals.openedition.org/essais/302

DOI : 10.4000/essais.302

ISSN : 2276-0970

\section{Éditeur}

École doctorale Montaigne Humanités

Édition imprimée

Date de publication : 1 juin 2018

Pagination : $39-49$

ISBN : 979-10-97024-06-2

ISSN : $2417-4211$

\section{Référence électronique}

Marine Totozani et Sandra Tomc, «Pluralité linguistique en classe de français langue seconde : quid de la variation? », Essais [En ligne], 14 | 2018, mis en ligne le 01 décembre 2019, consulté le 12 décembre 2019. URL : http://journals.openedition.org/essais/302 ; DOI : 10.4000/essais.302 


\section{Pluralité linguistique en classe de français langue seconde : quid de la variation? \\ Des représentations aux attitudes des enseignant.e.s}

Marine Totozani, Sandra Tomc

En nous appuyant sur les travaux menés en sociolinguistique et didactique des langues sur la pluralité linguistique et culturelle et la variation sociolinguistique, nous nous proposons, dans cet article, d'étudier la place de la variation sociolinguistique en classe de français langue seconde'. Ici, c'est le point de vue didactique que nous faisons prévaloir, même si les apports de la sociolinguistique restent incontournables.

Les questions qui ont présidé à cet article sont nées sur le terrain d'une recherche-action-formation (RAF) portant sur la prise en compte et la valorisation de la pluralité linguistique et culturelle en classe de français langue seconde dans des établissements scolaires des $1^{\text {er }}$ et $2^{\text {nd }}$ degrés à Saint-Étienne. Dans le cadre de cette recherche, des observations de classes menées dans le but de repérer les pratiques enseignantes à succès en ce sens ont dévoilé en même temps l'existence d'une vision parfois très " normée " de cette pluralité linguistique et culturelle se limitant souvent aux « langues » de l'élève et aux " langues " étrangères enseignées dans l'établissement. Ce constat a été le déclencheur d'un questionnement sur la place de la variation en classe de français langue seconde : quelle " conscience » de la variation les enseignant.e.s de français langue seconde développent-ils/elles ? quelle est leur perception de la pluralité linguistique et de la place qu'y occupe la variation ? que disent leurs discours sur ces phénomènes (socio)linguistiques vis-à-vis desquels ils/ elles manifestent une certaine frilosité dans leurs pratiques?

1 Cet article est issu en partie d'une communication présentée par Marine Totozani et Sandra Tomc : "Pluralité linguistique en classe d'accueil : quelle place pour la variation ? " au colloque Pluri-l, "Contributions au développement de perspectives plurilingues en éducation et formation ", Nantes, 2014. Par ailleurs, nous avons préféré abandonner l'acronyme UPE2A, plutôt opaque et un peu trop technique, au bénéfice de "classe de français langue seconde " qui permet par ailleurs de mieux situer notre recherche. 


\section{«L'arc en ciel de nos langues. Jalons pour une école plurilingue »}

La recherche-action-formation "L'arc-en-ciel de nos langues. Jalons pour une école plurilingue " poursuivait deux objectifs principaux : il s'agissait d'abord de repérer les pratiques pédagogiques et didactiques à succès d'enseignant.e.s recevant dans leurs classes des enfants issus de l'immigration dans le but de les formaliser, puis de les diffuser auprès du plus grand nombre. L'émergence des nouvelles vagues migratoires et la complexité linguistique qui les accompagne peuvent s'avérer parfois déconcertantes pour les enseignant.e.s, ce qui renforçait ce besoin de partage. C'était aussi un geste de reconnaissance de la passion et de l'énergie que déployaient tou.te.s ces enseignant.e.s désireux.euses de faire de leurs classes de véritables pépinières de la mise en ouvre d'une pédagogie inclusive. Ce travail contribuerait en même temps à faire évoluer les représentations des enseignant.e.s des $1^{\text {er }}$ et $2^{\text {nd }}$ degrés sur l'intégration des élèves allophones en classe ordinaire. L'ouvrage issu de cette recherche en est le reflet ${ }^{2}$.

Quant à la thématique de la variation, à travers le recueil des discours des enseignant.e.s sur ce sujet, notre objectif était d'obtenir des éléments de réponse par rapport à ce que les enseignant.e.s croient ou pensent à ce propos, mais aussi sur le rôle que la didactique des langues et l'école peuvent exercer sur les pratiques pédagogiques, sur les apprentissages.

\section{"Pluralité linguistique » et "variation » en sociolinguistique et en didactique des langues}

\section{Pluralité linguistique et variation sociolinguistique : quels rapports?}

Avec Véronique Castellotti, nous allons « envisager la pluralité linguistique dans une forme de globalité [...] [en la concevant] aussi bien entre les langues diverses qu'à l'intérieur d'une même "langue ", voire les deux à la fois ${ }^{3}$ ". De ce point de vue, un contexte de pluralité linguistique s'avère beaucoup plus complexe qu'une simple cohabitation de plusieurs langues. Il conviendrait, par conséquent, parler plutôt de "françaiS " au pluriel que de "la " langue française à enseigner et à apprendre.

L'organisation interne de cette pluralité linguistique n'est pas moins complexe: " envisager la pluralité linguistique dans une forme de globalité, implique [...] de s'intéresser davantage aux relations qu'aux langues elles-mêmes ${ }^{4}$ ». Or, nonobs-

2 Isabelle Graci, Marielle Rispail et Marine Totozani, (éds), L'arc-en-ciel de nos langues. Jalons pour une école plurilingue, Paris, L’Harmattan, 2017.

3 Véronique Castellotti, "Réflexivité et pluralité/diversité/hétérogéneité : soi-même comme des autres ? ", in Didier De Robillard (éd.), "Réflexivité, herméneutique. Vers un paradigme de recherche ? ", Cahiers de Sociolinguistique $\mathrm{n}^{\circ}$ 14, Rennes, Presses universitaires de Rennes, 2009, p. 132.

4 Véronique Castellotti, ibid. 
tant ces aller-retours qui caractérisent la vie des langues, le pouvoir politique et par conséquent l'école en ont de tout temps décidé autrement, en privilégiant certaines formes au détriment des autres, comme nous le rappellerons dans le paragraphe qui suit.

\section{Langue(s), norme(s), variations et représentations}

L'intérêt pour la variation dans le cadre de l'enseignement/apprentissage $\mathrm{du}$ français langue maternelle, étrangère ou seconde n'est pas récent ${ }^{5}$. Alors que la norme en classe de français langue seconde fait penser avant tout au "bon français " ${ }^{6}$, la variation consiste en la prise en compte des multiples réalisations langagières au sein d'un groupe social. La forte adhésion à une idéologie proche du purisme ne permet pas toujours le constat de la diversité. Pourtant la "langue ", concept majeur (et en même temps remis en cause) de la sociolinguistique, en opposition avec la vision structurale des langues de Saussure, est un fait social, non-systématique, hétérogène et plurinormé7. L'identification des variétés et de leur hiérarchisation est déterminante pour repérer les représentations des locuteur.trice.s, dans notre cas des enseignant.e.s, matérialisées dans des discours. Rappelons que par représentations, nous entendons une forme de connaissance, socialement élaborée et partagée, ayant une visée pratique ${ }^{8}$. Notre réflexion s'inspire des typologies de Françoise Gadet ${ }^{9}$, de nature sociolinguistique, et de Daniel Coste et $a l i i^{10}$, à la fois sociolinguistique et didactique, et de ce fait, davantage adaptée au contexte de la classe.

5 Citons, par ordre chronologique, les deux numéros de la revue Repères: «Ils parlent autrement : pour une pédagogie de la variation langagière " en 1985, puis "Éléments pour une didactique de la variation langagière » de 1988 ; Stéphanie Costa-Galligani et Cécile Sabatier, " La cour de récréation : zone d'intersection linguistique entre les quartiers et l'école ", in LouisJean Calvet et Auguste Moussirou-Mouyama (éds), Le plurilinguisme urbain, Actes du colloque international "Les villes plurilingues", Québec, Institut de la francophonie, 2000, p. 357-368; Josiane Boutet et Françoise Gadet, "Pour une approche de la variation linguistique ", in Le français aujourd'hui, n 143, 2003, p. 41-44 ; Françoise Gadet, "Quelle place pour la variation dans l'enseignement du français langue étrangère et seconde ? ", in Pré-textes franco-danois, Université de Roskilde, 2004, <halshs-00114390>, qui l'aborde du point de vue de la sociolinguistique ; plus récemment, les travaux réunis par Laurence Buson et Emmanuelle Guérin, dans le numéro 50 de Lidil «Variation stylistique et diversité des contextes de socialisation. Enjeux sociolinguistiques et didactiques ", 2014 ; et plus récemment encore l'article de Lucile Cagnon, Laurence Buson et Cyril Trimaille, "Développer la souplesse stylistique intra et interlinguistique : exploration d'une approche croisée en classe de CP-CE1 ", in Carnets D'atelier De Sociolinguistique, 2015.

6 Voir à ce propos Évelyne Charmeux, Le "bon français » et les autres, Toulouse, Éd. Milan, 1989.

7 William Labov, "Hypercorrection by the lower middle-class as a factor in linguistic change ", in William Bright, (éd.), Sociolinguistics, Mouton, 1966.

8 Denise Jodelet, Folies et représentations sociales, Paris, Les Presses universitaires de France, 1989.

9 Françoise Gadet, La variation sociale en français, Paris, Ophrys, 2003.

10 Daniel Coste, (éd.), Les langues au cour de l'éducation. Principes, Pratiques, Propositions, Fernelmont, E.M.E. Éditions, coll. Proximités Sciences du Langage, 2013. 


\section{Du terrain au terrain}

Le choix de mener une recherche-action-formation pour développer la prise en compte et la valorisation du plurilinguisme en classe de français langue seconde est lié au caractéristiques de cette recherche : "rechercheaction-formation collaborative avec des enseignants, en partant de leurs préoccupations, en co-construisant pas à pas avec eux une réflexion sur les représentations sociolinguistiques et leurs conséquences et en les accompagnant pour qu'émergent d'autres façons de travailler la langue de scolarisation ${ }^{11}$ et plus globalement, la pluralité des langues présentes en classe. Notre méthodologie combine observations de classe et entretiens semi-directifs effectués lors de la phase initiale de cette recherche avec les enseignant.e.s qui y ont participé et d'autres enseignant.e.s volontaires. Les réponses obtenues nous ont permis de récolter leurs discours sur la variation. Nous avons choisi d'y repérer le contenu et la place que les enseignant.e.s attribuaient à la variation dans leurs cours (pratiques déclarées), ce qui nous a permis de développer nos réflexions sur la place de la variation en classe de français langue seconde.

\section{«Conscience » de la variation?}

En appliquant à notre cas la notion de "conscience linguistique " développée par Eric Hawkins pour désigner "l'importance pour enseignants et éducateurs, d'une bonne connaissance de la nature et du fonctionnement du langage ${ }^{12}$ ", peut-on parler d'une " conscience de la variation" chez les enseignant.e.s ? Le développement d'une certaine conscience de la pluralité linguistique ${ }^{13}$, va-t-il de pair avec une conscience de la variation ? Quelles sont les représentations des enseignant.e.s sur la variation ? ${ }^{14}$

\section{Discours et représentations des enseignant.e.s sur la variation : entre reconnaissance et méconnaissance}

La reconnaissance de la pluralité linguistique en classe sous l'angle de la variation est difficile à concevoir. Pluralité linguistique et variation sont le plus

11 Stéphanie Clerc et Claude Richerme-Manchet, "La recherche-action-formation : une stratégie glottopolitique en terrain scolaire ", in Romain Colonna (éd.), Des paroles, des langues et des pouvoirs, Paris, L'Harmattan, 2014, p. 135.

12 Marie-Thérèse Vasseur, "Présentation ", in Michel Candelier, Gina Ioannitou, Danielle Omer et Marie-Thérèse Vasseur, Conscience du plurilinguisme, Rennes, Presses universitaires de Rennes, 2008, p. 9.

13 Michel Candelier, Gina Ioannitou, Danielle Omer et Marie-Thérèse Vasseur, ibid.

14 Voir à ce propos les travaux de Hélène Romian, "Quel(s) traitement(s) pédagogique(s) de la variation et des normes langagières ? " in Jacques Treigner et Hélène Romian (éds), Ils parlent autrement. Pour une pédagogie de la variation langagière, Repères, $\mathrm{n}^{\circ}$ 67, INRP, 1985. Jacques Treignier, Agnès Meray, "I parlent pas bien français, les Arabes. Normes évaluatives des enfants et des enseignants à l'école primaire ", in Jacques Treigner et Hélène Romian (éds), Ils parlent autrement. Pour une pédagogie de la variation langagière, Repères, $\mathrm{n}^{\circ}$ 67, INRP, 1985. 
souvent perçues séparément. La pluralité linguistique est envisagée comme une présence de plusieurs langues en classe, et malgré une certaine reconnaissance de la pluralité interne à la langue de scolarisation et aux autres langues de la classe, la place cette dernière au sein de cette pluralité plus large semble discutable. Par ailleurs, si la pluralité est souvent reconnue d'emblée comme une richesse, les phénomènes variationnels font l'objet de jugements de divers ordres que nous essaierons d'analyser ci-après.

Mais quelle(s) ouverture(s) sur la variation linguistique en classe de français langue seconde? Peut-on repérer, dans les discours des enseignant.e.s une acceptabilité de la variation ? Le français "légitimé » est souvent convoqué par les enquêté.e.s, comme cela apparait à travers les propos de l'une des enseignantes interrogées $^{15}$ :

moi je confonds le é et le è // je viens du sud // je leurs dis / faites pas comme moi / écoutez bien les gens de Saint-Étienne (B).

Dans cet exemple, la variation phonique est saillante. Elle renforce la représentation d'une nécessité de bien prononcer, contextualisée par l'axe géographique. Le régiolecte se noie sous le poids de la norme et dévoile une forme d'insécurité puisque la locutrice considère sa propre pratique linguistique comme non conforme à l'idée qu'elle se fait de cette norme ${ }^{16}$.

Malgré ce constat, on peut déceler une certaine conscience de la variation dans ses aspects phonologiques et lexicaux et moins dans celui syntaxique. Cette idée se renforce à travers les discours des autres enquêté.e.s, comme la locutrice A, pour laquelle cette conscience s'actualise dans le fait qu'il est interdit de formuler des jurons au sein de l'école :

parce que moi / ils m'entendent parfois dire merde et ça m’échappe / et je leur explique qu'est ce qui faut dire à la place // est ce que vous avez des mots pour remplacer ce vilain mot que je viens de dire / alors on dit zut flûte mince (A).

La variation est investie alors d'une valeur symbolique puisque l'enseignante en question choisit de se conformer à une norme situationnelle. Rappelons, à cet effet, avec Philippe Blanchet que

"l'acceptabilité d'un usage linguistique ne se définit pas dans l'absolu et pour lui-même, mais de façon sociale en fonction de paramètres contextuels nombreux ; c'est donc dans la prise en compte de la pluralité possible et sa confrontation avec un contexte précis et un objectif de relation que les choix d'acceptabilité ou non (car on peut jouer la contre-norme) se font ${ }^{17}{ }^{17}$

15 Conventions de transcription : (A) identification des locuteurs ; CAPITALE : intonation forte / pause courte // pause plus de deux secondes /// pause plus de cinq secondes.

16 Louis-Jean Calvet, Pour une écologie des langues du monde, Paris, Plon, 1999.

17 Philippe Blanchet, "Post-face en forme de coup de gueule : pour une didactisation de l'hétérogénéité linguistique contre l'idéologie de l'enseignement normatif et ses discriminations glottophobes ", in Valentin Feussi, Mylène Eyquem-Lebon, Auguste Moussirou-Mouyama et Philippe Blanchet, (éds), « Hétérogénéité sociolinguistique et didactique du français. Contexte 
Cependant, il est intéressant de se pencher sur les termes auxquels les enseignant.e.s ont recours pour désigner les phénomènes variationnels afin de mieux peaufiner le profil de cette conscience de la variation. D'un.e enseignant.e à l'autre, le flou s'installe au niveau des dénominations utilisées : "registre ", " niveau ", " langage » sont les termes qui reviennent le plus souvent :

pour les salutations / je pars de leurs mots à eux d'abord // les mots qu'ils utilisent eux / eux ils font pas la distinction entre le langage familier et le langage standard (A); moi / je préfere parler de niveau de langue / parce que registre au lycée / ça va être autre chose (B).

Confusion ou méconnaissance ? ou tout simplement une vision imprécise de la variation sociolinguistique?

\section{De quelle(s) variation(s) parle-t-on?}

Les enseignant.e.s ont une certaine conscience de la variation en français et de ce fait, ils/elles reconnaissent des sphères d'influence propres à la langue dite standard et à la variation comme il ressort des propos de l'enquêté suivant à deux moments de l'entretien :

dans la communication / dans l'oral qu'ils apprennent en dehors de nous / c'est dans les interactions avec les copains dans la cour etc. (A) ;

eux / ils font pas la distinction entre le langage familier et le langage standard (A).

Pour lui, dans le cas des élèves allophones, la difficulté majeure semble tenir au fait qu'ils/elles doivent vivre entre deux cultures : la culture d'origine, de la famille d'un côté et la culture du pays d'accueil, de l'école de l'autre côté, comme l'atteste l'extrait suivant :

et surtout dans le cercle familial / parce que c'est pris comme une espèce de distance / au contraire c'est pris comme à l'inverse de ce que nous on veut / de ce qu'on ressent // tu mets une distance entre les gens qui t'ont fait passer le sel ou le pain et eux ils prennent comme si t'étais des étrangers du coup (A) ; non / c'est pas qu'ils sont impolis // c'est que dans leur culture on dit pas s'il te plait et merci à tout bout de champ (A).

Cette conscience de la variation est aussi relevée en langue et culture d'origine notamment par le biais d'usages situés, comme dire «bonjour » en arabe :

et par exemple / quand tu parles avec des arabophones // comment on dit bonjour en arabe // la plupart des enfants / ils vont te dire salamalekum // je leur dis / mais enfin les enfants y a quand même d'autres façons de saluer quelqu'un que de dire salamalekum / y quand même une connotation religieuse (A).

francophones plurilingues ", Cahiers de Linguistique n 35/2, 2009, p. 165-183. 
La variation se manifeste aussi sous le paradigme du genre ${ }^{18}$. Les thématiques genrées semblent particulièrement prégnantes en classe de français langue seconde ${ }^{19}$. De ce fait, comme cela a souvent été le cas, la classe de français langue seconde peut être considérée une nouvelle fois comme une loupe par rapport à la classe ordinaire. L'usage linguistique apparait comme un marqueur identitaire déterminant, l'établissement de deux groupes distincts [les deux pôles que sont l'homme et la femme entrainant] un effet d'organisation sous forme d'opposition ${ }^{20}$ :

un élève qui va dire une meuf / c'est un provocateur lui /// je lui ai dit que c'était pas adapté / je lui ai fait répéter / j'ai dit pardon / j'ai // j’ai pas bien compris ce que tu as dit / ben il a répété / alors je luis dis simplement / non je pense que c'est pas adapté ce que tu dis (B).

\section{Des représentations aux attitudes : un tableau en clair-obscur}

Avant de passer de l'étude des représentations à l'étude des attitudes, nous allons définir ces dernières comme une dimension évaluative des représentations sociales ${ }^{21}$. Il s'agit donc de saisir la "position (plus ou moins cristallisée) d'un agent (individuel ou collectif) envers un objet (personne ou groupe, situation ou événement) $»^{22}$. Ici, nous cherchons à circonscrire les attitudes enseignantes à l'égard de la variation au travers des jugements émis par les enseignant.e.s qui sous-tendent jusqu'à une certaine mesure leurs pratiques de classes. Nous y parviendrons à l'aide du repérage d'attributs qui nous permettent de formuler l'hypothèse selon laquelle on pourrait considérer ces attitudes comme un tableau en clair-obscur dans lequel se croisent notes optimistes et moins optimistes.

Ainsi, à côté d'attitudes traduisant une certaine tendance de déni voire de dénigrement vis-à-vis de la variation sociolinguistique en classe, nous avons pu repérer des attitudes d'ouverture et même d'efforts allant dans le sens d'une didactisation.

18 Sandra Tomc et Grâce Ranchon précisent que le genre est un marqueur privilégié pour identifier les processus relationnels et les rapports de pouvoir. Les études sur le genre s'intéressent à la manière dont se composent pour les êtres humains, le féminin et le masculin, comment s'organisent les relations sociales, les rapports de pouvoir et les hiérarchies entre les femmes et les hommes, voire à la déconstruction du paradigme de la bicatégorisation. «Identités/marquage/ genre ", in Marielle Rispail (éd.), Abécédaire de sociodidactique - 65 notions et concepts, SaintÉtienne, Presses Universitaires de Saint-Étienne, 2017, p. 60-61.

19 Ces thématiques ont déjà été abordées entre autres dans un article co-écrit par Sandra Tomc et Marine Totozani, « Être fille ici et là-bas ", in Sandra Tomc, Marine Totozani, Grâce Ranchon et Mireille Baurens (éds), "Genres Langues et Pouvoirs ", Cahiers de Linguistique. Revue de sociolinguistique et de sociologie de la langue française, Fernelmont, E.M.E. Edditions, 2013.

20 Sandra Tomc, Sophie Bailly et Grâce Ranchon, (éds), Pratiques et Langages du genre et du sexe : déconstruire l'idéologie sexiste du binarisme, E.M.E. Éditions, Collection Proximités, 2016, p. 10.

21 Pascal Molinier, Images et représentations sociales, Grenoble, Presses Universitaires de Grenoble, 1996.

22 Gilles Ferreol, "Attitude ", in Gilles Ferreol et Guy Jucquois (éds), Dictionnaire de l'altérité et des relations interculturelles, Paris, Armand Colin, 2003, p. 34-35. 


\section{Déni, dénigrement?}

Même si les données recueillies ne nous permettent pas de généraliser les attitudes de déni de la variation, celles-ci se manifestent parfois à travers l'attachement à une norme linguistique idéale, proche de l'écrit. L'autre versant de cette attitude conduit l'enquêté dans l'exemple qui suit, à nier l'existence d'une dimension communicationnelle :

euh // non mais / y en a un qui a dit ça m’intéresse pas // alors je lui dis / ça ne m’intéresse pas $(\mathrm{C})$.

En reprenant son élève : ça ne m’intéresse pas, l'enseignant en question légitime la norme écrite au détriment de l'oral alors qu'il emploie lui-même ça au lieu de cela. Dans la suite de l'exemple, la particule adverbiale ne pas n'est pas actualisée dans c'est pas dit, ils ont pas de maison, ils sont pas censés :

y a des roms qui parlent le langage de la rue comme par exemple j'm'en fous fiche moi la paix / c'est pas dit méchamment / ils vivent dans la rue ils ont pas de maison donc ils parlent le langage de la rue / ils sont pas censés le savoir je leur dis c'est des bêtises tu peux pas parler comme ça (C).

Notons que paradoxalement, $\mathrm{C}$ est dans la variation quand il utilise $y$ a pour il y a, c'est des bêtises au lieu de ce sont. Il accepte néanmoins le "langage de la rue " mais le qualifie de bêtises en ne contextualisant pas ses propos et ne fournissant pas d'explicitations par rapport aux usages sociaux. C'est la langue légitimée qui est souvent mise en en avant :

j'accorde de l'importance au français standard // ils doivent être capables de se débrouiller à la cantine / dans la rue / on fait des sketches / des mimes de situation / par exemple le Petit Chaperon Rouge on mime le bonjour grand-mère (C).

mais norme versus variation sont parfois abordées de pair aussi, même si timidement :

ce que je fais / je leur dis / je leur dis // écoutez // enfin // pour ceux qui sont un peu plus curieux / bon / vous savez qu'il y a plusieurs niveaux de langue // dans votre pays / dans toutes les langues il y a des niveaux de langue / je vous le dis / je vous le dis / en tant qu'enseignante / voilà ce qu'on dit en langage soutenu / je préfère que vous le sachiez / (...) je le fais / et sans avoir honte quoi // non / non / je le dis / sans me dire qu'il faut pas (B).

\section{Des ouvertures?}

Connaitre une langue c'est en connaitre ses usages langagiers et savoir s'adapter aux situations de communication variées pour lesquelles elle est sollicitée. C'est le cas de A qui invite ses élèves à mener une enquête en famille sur leurs répertoires plurilingues, suivie d'un moment de réflexion en classe :

et je dis / ben // demandez à vos parents comment on peut dire bonjour autrement que par Salamalekum /// allez interroger vos parents / et en arabe y a un autre mot y a que je prononce mal mais un mot du type Meraba euh qui veut dire bonjour (A). 
ou qui les sensibilise aux usages sociaux de la langue :

qu'ils prennent conscience que c'est des choses qu'on peut pas dire avec n'importe qui n'importe où / dans n'importe quelles circonstances / on essaie à un moment donné de pouvoir en parler parce qu'il faut bien le mettre sur la table (A);

j'accorde de l'importance au français standard car je sais trop que les mots qu'ils vont utiliser vont avoir des connotations sociales TERRIBLES (A).

\section{Vers une didactisation de la variation?}

De quelle part de liberté, les enseignant.e.s bénéficient-ils/elles pour enseigner la variation? Subissent-ils/elles une pression institutionnelle pour enseigner ou a contrario refouler la variation sociolinguistique en classe?

y a le $\mathrm{BO} /$ nous on est FLSco // c'est tellement vague // on est complètement tranquille / l'inspecteur ne s'y connait pas // il me l'a dit // c'est vous les professionnels / moi je regarde sur internet je suis libre (B).

A priori non, sauf pour le DELF où on revient à la langue normée / légitimée :

je suis obligée parfois de garder les élèves deux ans dans ma classe /// c'est à cause du DELF (B).

Pour amener les enquêté.e.s vers l'idée de la didactisation de la variation sociolinguistique, l'entretien s'est révélé un véritable outil de formation des enseignant.e.s. Les échanges entre enquêtrice et enquêté.e.s ont déclenché un travail réflexif chez ces derniers. Ainsi, dans l'extrait qui suit, au début, l'enseignante affirme ne pas avoir fait de séquence sur ça / si je l'ai fait une fois (B) avant de déclarer que finalement ah / mais c'est pas une mauvaise idée finalement que de prévoir des séquences sur la variation :

(B) dans mon enseignement tu veux dire / j'accorde de l'importance aux registres / je suis obligée / c'est dans les programmes /// il faut que les élèves connaissent les niveaux de langue / mais je ne consacre pas probablement de séquence sur ça // si / je l'ai fait une fois / une séquence à propos de l'interrogation où j'avais travaillé une fois sur les niveaux de langue / tu vois / familier / standard / soutenu euh ;

(Enquêtrice) d'habitude donc / tu ne fais pas d'activités plus précises que ça euh //;

(B) je pourrais, je pourrais /// ah / mais c'est pas une mauvaise idée finalement.

\section{Des pratiques à formaliser et à diffuser}

Le degré de prise de conscience de la variation s'accroit au fur et à mesure de l'enquête. A explique faire seulement des commentaires :

jamais le laisser passer sans qu'il y ait un commentaire dessus // les ta gueule / fous le camp (A)

avant finalement d'affirmer pouvoir se réappuyer là-dessus, l'idée étant de faire quelque chose avec : 
dans la communication / dans l'oral qu'ils apprennent en dehors de nous // c'est dans les interactions avec les copains dans la cour etc. / là où ils font très vite des progrès c'est dans les insultes // après / en classe il faut pouvoir se réappuyer là-dessus $(\mathrm{A})$.

De fait, l'essai de didactisation de la variation à travers les albums favorise largement l'entrée dans la langue de scolarisation :

j'essaie, à travers certains albums de traiter des insultes // "La princesse grenouille » / princesse transformée en grenouille qui insulte / elle le traite de goinfre / d'idiot / qu'il connait pas les manières / de sauvage (A).

\section{Quid de la variation en classe de français langue seconde?}

La réponse s'avère nuancée : si les enseignant.e.s développent une certaine conscience de la variation, leur conceptions de la variation et la terminologie utilisée pour la désigner sont parfois floues et imprécises. De ce fait, si la pluralité linguistique est perçue comme une richesse à développer, des tensions se révèlent dans les attitudes à l'égard de la variation : celle-ci est tantôt ignorée, voire rejetée comme impure, tantôt reconnue et exploitée.

À quoi peut-on imputer cet état de fait : à une insuffisante prise en compte de la variation dans les programmes d'enseignement des langues en France, dont le français ? à la formation des enseignant.e.s ? au poids de la norme ?

Depuis plus de vingt ans déjà, sociolinguistes et didacticien.ne.s insistent sur l'importance de la prise en compte la variation en classe de langue. Si l'on considère la diversité comme base fondamentale de l'humain [...] qui se matérialise dans des formes de pluralité et d'hétérogénéitée ${ }^{23}$, ne devrait-on pas se soucier davantage de la place de la variation dans la formation des enseignant.e.s de langue?

Marine Totozani

CELEC (EA 3069)

Univ Lyon UJM Saint-Étienne marine.totozani@univ-st-etienne.fr

Sandra Tomc CELEC (EA 3069)

Univ Lyon UJM Saint-Étienne sandra.tomc@univ-st-etienne.fr

23 Véronique Castellotti, «Diversité(s), histoire(s), compréhension... Vers des perspectives relationnelles et alterdidactiques pour l'appropriation des langues ", in David Bel et Emmanuelle Huver, "Prendre la diversité au sérieux en didactique / didactologie des langues ", Recherches en didactique des langues et des cultures Les cahiers de l'Acedle, 12-1, 2015, p. 3-4. 


\section{Résumé}

L'existence d'une conscience de la pluralité linguistique chez les enseignant.e.s de français langue seconde serait-elle exclusive d'une conscience de la variation intralinguistique comme partie constitutive de cette pluralité ? Il se nourrit des travaux menés en sociolinguistique et didactique des langues sur le plurilinguisme et la variation (socio)linguistique. Les réflexions développées ici prennent appui sur les résultats d'une enquête effectuée lors d'une rechercheaction-formation portant sur la valorisation de la diversité linguistique et culturelle dans les $1^{\text {er }}$ et $2^{\text {nd }}$ degrés à Saint-Étienne.

\section{Mots-clés}

Pluralité linguistique, variation, enseignant.e.s de français langue seconde, représentations, attitudes.

\section{Abstract}

French as a Second Language teachers are aware of linguistic plurality, but runs this counter to them being aware of intralinguistic variation as a constitutive part of this very plurality? The analysis is grounded on existing work on language variation and plurilingualism in sociolinguistics and language education/didactics. The data was collected through a study articulating an actionbased research and a training session promoting linguistic and cultural diversity in primary and secondary schools in Saint-Étienne, France.

\section{Keywords}

Linguistic plurality, variation, French as a Second Language teachers, representations, attitudes. 